\title{
Association Between Androgenic Alopecia and Coronary Artery Disease: A Cross-Sectional Study of Han Chinese Male Population
}

\author{
Ya-Xin Wang (D) \\ Xiao-Wen Chen \\ Si-Bo Wang \\ Ling-Feng Gu \\ Ya-Fei Li \\ Yao Ma \\ Hao Wang \\ Lian-Sheng Wang
}

Department of Cardiology, Jiangsu Province People's Hospital and Nanjing Medical University First Affiliated Hospital, Nanjing, 210029, People's Republic of China
Correspondence: Lian-Sheng Wang Department of Cardiology, Jiangsu Province People's Hospital and Nanjing Medical University First Affiliated Hospital, 300 Guangzhou Road, Nanjing, 210029, People's Republic of China $\mathrm{Tel} / \mathrm{Fax}+862583724440$

Email drlswang@njmu.edu.cn
Purpose: This research aimed to investigate the correlation between androgenic alopecia (AGA) and coronary artery disease (CAD) and analyze its value in predicting the severity of coronary atherosclerosis in the Han Chinese male population.

Patients and Methods: A total of 402 Han Chinese male patients aged 28-75 years were enrolled and performed coronary angiography (CAG) after admission. According to the BASP classification, the participants were divided into mild, moderate and severe AGA. CAD was determined via $\mathrm{CAG}$ and defined as stenosis of $\geq 50 \%$ in at least one major coronary artery, and the Gensini score was calculated to evaluate the severity of coronary atherosclerosis.

Results: In this study, CAD status $(P=0.002)$, dyslipidemia status $(P=0.002)$, age $(P=$ $0.003)$ and coronary atherosclerosis severity $(P<0.001)$ were different in patients with different levels of AGA. Multivariate logistic regression analysis revealed that severe AGA was independently correlated to CAD risk (OR, 2.111; 95\% CI 1.152 to $3.870, P=0.016$ ), while the relative CAD risk of early-onset AGA was 2.292 (OR, 2.292; 95\% CI 1.132 to 4.640, $P=0.021)$. AGA status (OR, 2.247; $95 \%$ CI 1.396 to $3.617, P=0.001)$, severe AGA (OR, 2.360; 95\% CI 1.506 to 3.699, $P<0.001$ ) and early-onset AGA (OR, 3.474; 95\% CI 2.069 to $5.832, P<0.001)$ were all independently associated with the severity of coronary atherosclerosis. The area under the receiver operating characteristic (ROC) curve plotted using severe AGA was 0.601 , which is predictive of severe coronary atherosclerosis. Moreover, the presence of severe AGA increases the risk of developing CAD associated with obesity $(\mathrm{SI}=1.663, \mathrm{SIM}=1.222, \mathrm{AP}=0.289)$, diabetes $(\mathrm{SI}=2.239, \mathrm{SIM}=1.149, \mathrm{AP}$ $=0.503)$ and dyslipidemia $(\mathrm{SI}=1.062, \mathrm{SIM}=0.646, \mathrm{AP}=0.045)$.

Conclusion: This study suggested that AGA is independently associated with CAD in a Han Chinese male population. AGA may be a simple and feasible method for screening $\mathrm{CAD}$ and indicative of the severity of coronary atherosclerosis.

Keywords: coronary artery disease, coronary atherosclerosis, androgenic alopecia, coronary angiography, cardiovascular disease

\section{Introduction}

Coronary artery disease (CAD) is the primary factor of death in most developing or developed countries; ${ }^{1}$ the prevalence of CAD has put enormous pressure on public health. ${ }^{2}$ To reduce the medical burden caused by CAD, simple and reliable signs for the identification of CAD are urgently needed.

Androgenic alopecia (AGA) is of the highest prevalence in all types of hair loss. It affects both genders and different races with different patterns and prevalence of 
hair loss. ${ }^{3}$ Over the past several decades, a few researches have suggested that AGA may be correlated to CAD and established cardiovascular disease (CVD) risk factors such as diabetes, hypertension and dyslipidemia. ${ }^{4-9}$ Serum free androgens have been thought to play an important part in the etiopathogenesis of AGA, which can bind to androgen receptors on the scalp to micronize hair follicles leading to hair loss. ${ }^{10}$ It has been reported that serum-free androgens may stimulate vascular smooth muscle cells (VSMC) proliferation to promote atherosclerosis and cause vasoconstriction leading to hypertension. ${ }^{11}$ Insulin resistance, as a CAD risk factor, can aggravate AGA, which may explain the positive correlation between AGA and CAD. ${ }^{12,13}$ Chronic inflammation has been thought to play an important role in the occurrence and development of CAD and may also be involved in the association between AGA and CAD. ${ }^{12,14}$

Despite AGA affects different races with different patterns and prevalence of hair loss, most prior studies of the association between AGA and CAD were based on Caucasians. There is still insufficient research on the relevance of AGA to $\mathrm{CAD}$ in the Chinese population. Furthermore, the diagnosis and severity assessment of CAD in some studies were not based on the coronary angiography (CAG), resulting in a lack of reliability. This study aimed to investigate the relevance of AGA to CAG-certified CAD and CVD risk factors and analyze its value in predicting the severity of coronary atherosclerosis in the Han Chinese male population.

\section{Methods}

\section{Study Participants}

This cross-sectional study is based on the Han Chinese male population. From December 2020 to March 2021, 402 male patients aged 28-75 years were hospitalized in Nanjing Medical University First Affiliated Hospital, Nanjing (China) and performed CAG for suspected or confirmed CAD. The applicable inclusion criteria were: male individuals aged $\leq 75$ years. The exclusion criteria included: history of CAG or percutaneous coronary intervention (PCI) or coronary artery bypass grafting (CABG), valvular heart disease, endocarditis or cardiomyopathy, thyroid diseases or adrenal dysfunction, severe hepatic insufficiency, history of stroke, malignant tumor and other types of alopecia. This study was conducted after the approval of the Ethics Committee of the First Affiliated Hospital of Nanjing Medical University (2011-
SRFA-005). Our research was carried out in accordance with the Declaration of Helsinki. All participants subscribed informed consent.

\section{Coronary Angiography}

After Seldinger puncture of the patient's radial or femoral artery, two experienced coronary interventionists used Judkins technology to perform left and right coronary angiography (CAG), respectively. ${ }^{15}$ The interventionists assessed the degree of coronary stenosis based on the American Heart Association (AHA) method. ${ }^{16}$ Significant CAD was defined as stenosis of $\geq 50 \%$ in at least one major coronary artery. ${ }^{17}$ Gensini scoring system was used as the measurement of the severity of coronary atherosclerosis. The computing method of the Gensini score is on the basis of the number of coronary artery stenosis segments, including the geographical importance of the lesion and the degree of lumen stenosis. ${ }^{18}$

\section{Androgenetic Alopecia Criteria}

The severity of AGA was identified by the same dermatologist according to the BASP classification, which is a new, systematic and universally applicable classification method for AGA and recommended by the Chinese Society of Dermatology. ${ }^{19}$ The BASP classification is classified according to the hairline shape, forehead and top hair density, including 4 basic types (BA) and 2 special types (SP). Combining the special and basic types will give the final classification. ${ }^{20}$ The severity of AGA is divided into three levels according to the BASP classification: mild AGA includes L, M0, M1, M2, C0, C1; moderate AGA includes $\mathrm{M} 3, \mathrm{C} 2, \mathrm{~V} 1, \mathrm{~V} 2, \mathrm{~F} 1$ and F2; severe AGA includes C3, U1 U2, U3, V3, F3. Early-onset AGA was defined as having moderate or severe alopecia before the age of 40 .

\section{Demographic Data}

With the patient's consent, professionals uniformly trained by the first author used face-to-face interviews to collect data. Through the questionnaire survey, we obtained demographic information (age, smoking, drinking, exercise, and family history of CAD). The smoking status of the research objects was divided into never smoking or smoking (including both previous and current smoking). Drinking status was divided into never drinking and drinking (including both previous and current drinking). Subjects who exercised were defined as having more than three physical activities per week (walking, biking, 
running, swimming, etc.) for a total time of more than 150 minutes. The same physician measured the height and weight and then calculated the body mass index (BMI) which is the weight $(\mathrm{kg})$ divided by the square of height $\left(\mathrm{m}^{2}\right)$ to assess obesity.

\section{Blood Pressure Measurement}

The resting blood pressure of patients was measured by electronic sphygmomanometer three times, and the average of the measurement results was taken as the final data. Subjects with systolic blood pressure (SBP) $\geq 140 \mathrm{mmHg}$ or diastolic blood pressure (DBP) $\geq 90 \mathrm{mmHg}$ or treated with antihypertensive drugs were recorded as having hypertension.

\section{Laboratory Measurements}

After a 12-hour fast, blood samples were taken immediately before coronary angiography between 7:00 am and 8:00 am. An automatic analyzer (AU 2700 Olympus, 1st Chemical Ltd, Japan) was used for enzymatic detection to determine total cholesterol (TC, mmol/L), triglycerides (TG, mmol/L), low-density lipoprotein cholesterol (LDLc, mmol/L), high-density lipoprotein cholesterol (HDL-c, $\mathrm{mmol} / \mathrm{L})$, Lipoprotein a (Lp(a), mg/L) and fasting blood glucose (FBG, mmol/L). Glycosylated hemoglobin (HbA1c) was measured via chromatography using a glycosylated hemoglobin meter (Variant II TURBO glycosylated hemoglobin meter, BIO-RAD, the USA). Dyslipidemia was defined as having $\mathrm{TG} \geq 2.3 \mathrm{mmol} / \mathrm{L}$ $(200 \mathrm{mg} / \mathrm{dL})$ or $\mathrm{TC} \geq 6.2 \mathrm{mmol} / \mathrm{L}(240 \mathrm{mg} / \mathrm{dL})$ or $\mathrm{LDL}-\mathrm{c}$ $\geq 4.1 \mathrm{mmol} / \mathrm{L}(160 \mathrm{mg} / \mathrm{dL})$ or HDL-c $<1.0 \mathrm{mmol} / \mathrm{L}(40 \mathrm{mg} /$ $\mathrm{dL})$ or taking lipid-lowering drugs. ${ }^{21} \mathrm{FBG}>7.0 \mathrm{mmol} / \mathrm{L}$ $(126 \mathrm{mg} / \mathrm{dL})$ or $\mathrm{HbA} 1 \mathrm{c} \geq 6.5 \%$ or receiving hypoglycemic therapy was defined as diabetes.

\section{Statistical Analysis}

We analyzed the data using a social science statistical software package (V.25.0; SPSS Corporation, Chicago, Illinois, USA). Continuous variables are judged to be normal distribution by the K-S normality test. Normal distribution parameters were expressed as mean \pm SDs and compared by independent sample $t$ test or one-way analysis of variance (ANOVA). Skew data were expressed in median, and were compared using Mann-Whitney $U$-test or Kruskal-Wallis test. A $\chi 2$ analysis was performed to compare the categorical variables between the groups. The ORs concerning the presence of CAD and "high stenosis" were determined by univariate or multivariate logistic regression analysis, and were shown as $95 \%$ CIs.

To analyze possible positive or negative interactions of severe AGA with obesity, diabetes and dyslipidemia, the ORs, 95\% CI and two-tailed P values were calculated in a $4 \times 2$ table. It was assumed that individuals unexposed to established CAD risk factors and severe AGA have a standard CAD risk (OR00 is 1); OR10 referred to the relative risk for $\mathrm{CAD}$ among patients without severe AGA and exposed to established CAD risk factors compared to those with neither severe AGA nor exposure; OR01 referred to the relative risk of severe AGA patients who were unexposed to established CAD risk factors compared to those with neither severe AGA nor exposure; the ratio of CAD risk in exposed patients with severe AGA to those with neither severe AGA nor exposure was OR11. These ORs were used to calculate the synergy indexes. ${ }^{22}$

The sensitivity, specificity, predictive value of severe AGA in identifying severe coronary atherosclerosis and the area under the ROC curve were calculated. ${ }^{23}$

\section{Results}

\section{The Baseline Characteristics of the Participants}

The study included 402 male patients whose median age is 61 years old. The baseline characteristics of the subjects were presented in Tables 1 and 2 . The differences of age $(P=0.003)$, SBP $(P<0.001)$, DBP $(P=0.001)$, dyslipidemia $(P=0.002)$, HbA1c $(P=0.004), \mathrm{CAD}$ status $(P=0.002)$ and Gensini scores $(P<0.001)$ among participants with mild, moderate and severe AGA were statistically significant. The median Gensini score was used as the cut-off point, and the Gensini score $\geq 22$ was defined as "high stenosis". The prevalence of "high stenosis" was higher in patients with severe AGA $(P<0.001)$. Between CAD and non-CAD participants, significant differences existed in BMI $(P=0.045)$, diabetes $(P<0.001)$, dyslipidemia $(P<0.001)$, HDL-c $(P=0.003)$, glucose $(P<0.001)$, HbA1c $(P<0.001)$, severe AGA $(P=0.002)$ and Gensini scores $(P<0.001)$.

\section{Univariate Logistic Regression Analysis with Respect to the Presence of CAD}

A univariate logistic regression was used to analyze risk factors of CAD, and the results are shown in Table 3. The risk of CAD increased by $8.5 \%$ for every $1-\mathrm{kg} / \mathrm{m} 2$ increase in BMI (OR, 1.085; 95\% CI 1.002 to $1.176, P=0.046)$ in all objects. Additionally, participants with diabetes were about 
Table I Characteristics of the Participants with Mild AGA, with Moderate AGA and with Severe AGA

\begin{tabular}{|c|c|c|c|c|c|}
\hline Characteristic & $\begin{array}{l}\text { All Participants } \\
(n=402)\end{array}$ & $\begin{array}{l}\text { With Mild AGA } \\
(n=187)\end{array}$ & $\begin{array}{l}\text { With Moderate AGA } \\
(n=66)\end{array}$ & $\begin{array}{l}\text { With Severe AGA } \\
(n=149)\end{array}$ & $\mathbf{P}$ value \\
\hline Age (years) & 61 (28 to 75$)$ & 51 (28 to 74$)$ & 62 (38 to 74$)$ & 62 (39 to 75$)$ & 0.003 \\
\hline BMI $\left(\mathrm{kg} / \mathrm{m}^{2}\right)$ & $25.30 \pm 3.19$ & $25.20 \pm 2.97$ & $25.5 I \pm 3.5 I$ & $25.33 \pm 3.32$ & 0.782 \\
\hline SBP $(\mathrm{mmHg})$ & $128.43 \pm 18.44$ & $122.84 \pm 16.67$ & $125.91 \pm 19.79$ & $136.56 \pm 17.07$ & $<0.001$ \\
\hline $\mathrm{DBP}(\mathrm{mmHg})$ & $78.61 \pm 11.86$ & $76.85 \pm 11.44$ & $77.11 \pm 13.37$ & $81.84 \pm 11.16$ & 0.001 \\
\hline Hypertension, n(\%) & 291 (72.4\%) & I 36 (72.7\%) & 46 (69.7\%) & 109 (73.2\%) & 0.863 \\
\hline Diabetes, $n(\%)$ & $123(30.6 \%)$ & 56 (29.9\%) & $16(24.2 \%)$ & $51(34.2 \%)$ & 0.330 \\
\hline Dyslipidemia, n(\%) & 259 (64.4\%) & III (59.4\%) & $36(54.5 \%)$ & 112 (75.2\%) & 0.002 \\
\hline Smoking, n(\%) & $293(72.9 \%)$ & $138(73.8 \%)$ & $50(75.8 \%)$ & $105(70.5 \%)$ & 0.672 \\
\hline Drinking, n(\%) & $26 \mid(64.9 \%)$ & I2I (64.7\%) & 47 (7I.2\%) & $93(62.4 \%)$ & 0.458 \\
\hline Exercise, n(\%) & $292(72.6 \%)$ & $14 \mid$ (75.4\%) & 48 (72.7\%) & $103(69.1 \%)$ & 0.440 \\
\hline $\mathrm{TC}(\mathrm{mmol} / \mathrm{L})$ & $3.94 \pm 1.10$ & $3.90 \pm 1.09$ & $3.97 \pm 1.04$ & $3.96 \pm 1.15$ & 0.822 \\
\hline $\mathrm{TG}(\mathrm{mmol} / \mathrm{L})$ & $1.38(0.4 \mathrm{I}$ to 18.99$)$ & I.4I (0.49 to I4.8I) & $1.20(0.50$ to 8.18$)$ & I.38 $(0.4 \mathrm{I}$ to 18.99$)$ & 0.360 \\
\hline LDL-c(mmol/L) & 2.26 (0.79 to 5.05$)$ & 2.27 (0.87 to 4.40$)$ & 2.36 (0.97 to 3.78$)$ & 2.23 (0.79 to 5.05$)$ & 0.940 \\
\hline HDL-c(mmol/L) & 0.95 (0.56 to $2.4 I)$ & 0.95 (0.57 to 1.90$)$ & $\mathrm{I} .0 \mathrm{I}(0.56$ to $2.4 \mathrm{I})$ & 0.93 (0.60 to I.83) & 0.166 \\
\hline Lp(a)(mg/L) & 137 (I to II87) & 142 (2 to II87) & 120.5 (12 to 1137$)$ & 137 (I to 987$)$ & 0.674 \\
\hline $\mathrm{FBG}(\mathrm{mmol} / \mathrm{L})$ & $5.00(2.98$ to 15.19$)$ & $4.90(2.98$ to 15.19$)$ & 4.89 (3.36 to II .56$)$ & $5.13(3.54$ to 13.20$)$ & 0.083 \\
\hline $\mathrm{HbAlc}(\%)$ & $5.80(4.50$ to 13.00$)$ & $5.70(4.50$ to $1 \mathrm{I} .60)$ & 5.85 (4.80 to 9.80$)$ & $6.00(5.00$ to 13.00$)$ & 0.004 \\
\hline CAD, n(\%) & 321 (79.9\%) & 145 (77.5\%) & $45(68.2 \%)$ & |3| (87.9\%) & 0.002 \\
\hline Gensini scores & 22.0 (0.0 to I52.0) & 19.0 (0.0 to 124.0) & I 8.5 (0.0 to 102.0$)$ & 36.0 (0.0 to I52.0) & $<0.001$ \\
\hline High stenosis, n(\%) & 207 (5I.5\%) & 84 (44.9\%) & 26 (39.4\%) & 97 (65.1\%) & $<0.001$ \\
\hline
\end{tabular}

Note: The $P$ values represent the overall comparison among the three groups.

Abbreviations: CAD, coronary artery disease; BMI, body mass index; SBP, systolic blood pressure; DBP, diastolic blood pressure; AGA, androgenetic alopecia; TC, total cholesterol; TG, total triglyceride; HDL-c, fasting high-density lipoprotein cholesterol; LDL-c, fasting low-density lipoprotein cholesterol; Lp(a), lipoprotein(a); FBG, fast blood glucose.

3.4-fold more likely to develop CAD (OR, 4.406; 95\% CI 2.124 to $9.139, P<0.001)$ than those without diabetes. Moreover, participants with dyslipidemia were approximately 2.5-fold as likely to develop CAD (OR, 2.501; 95\% CI 1.523 to $4.108, P<0.001)$ as participants without dyslipidemia.

Compared with participants with mild AGA, participants with severe AGA were approximately twice as likely to have CAD (OR, 2.108; 95\% CI 1.156 to $3.843, P=0.015$ ). Additionally, compared to those without severe AGA, participants with severe AGA had a significantly increased risk of CAD (OR, 2.413; 95\% CI 1.366 to $4.263, P=0.002$ ). Moreover, participants with early-onset AGA were 1.441fold more likely to develop CAD (OR, 2.441; 95\% CI 1.235 to $4.824, P=0.010$ ) than those without early-onset AGA.

\section{Multivariate Logistic Regression Analysis with Respect to the Presence of CAD}

To determine the risk factors of CAD in all participants, a multivariate logistic regression analysis was performed. In Table 4, the results of the multivariate model are presented as adjusted ORs and 95\% CIs. After adjusting for BMI, age, hypertension, dyslipidemia, diabetes, smoking, alcohol consumption, exercise status, and family history of CAD, the relative risk of CAD in severe AGA patients was the highest (OR, 1.807 ; $95 \%$ CI 0.952 to $3.430, P=0.070$ ), despite the difference not being statistically significant. The association of severe AGA (OR, 2.111; 95\% CI 1.152 to $3.870, P=0.016$ ) with the risk of CAD remained independent among all participants. Nevertheless, after other CAD risk factors were adjusted, the intensity of association between severe AGA and CAD was lower than that of early-onset AGA, whose relative CAD risk was 2.292 (OR, 2.292; 95\% CI 1.132 to $4.640, P=0.021$ ).

\section{Multivariate Logistic Regression Analysis with Respect to the Presence of "High Stenosis"}

AGA status, severe AGA and early-onset AGA were, respectively, included in the multivariate Logistic regression model with "high stenosis" as the dependent variable (Table 5), and the results showed that the relative risk of "high stenosis" in participants with severe AGA was the highest in all levels of AGA (OR, 2.247; 95\% CI 1.396 to 
Table 2 Characteristics of CAD and Non-CAD Participants

\begin{tabular}{|c|c|c|c|}
\hline Characteristic & $\operatorname{CAD}(n=321)$ & Non-CAD $(n=8 I)$ & $P$ value \\
\hline Age (years) & 61 (28 to 75$)$ & 61 (33 to 74$)$ & 0.243 \\
\hline BMI $\left(\mathrm{kg} / \mathrm{m}^{2}\right)$ & $25.46 \pm 3.23$ & $24.66 \pm 2.96$ & 0.045 \\
\hline $\mathrm{SBP}(\mathrm{mmHg})$ & $|28.62 \pm| 8.23$ & $127.70 \pm 19.36$ & 0.691 \\
\hline $\mathrm{DBP}(\mathrm{mmHg})$ & $78.67 \pm 11.70$ & $78.37 \pm|2.5|$ & $0.84 I$ \\
\hline Hypertension, n(\%) & 235 (73.2\%) & $56(69.1 \%)$ & 0.464 \\
\hline Diabetes, $n(\%)$ & II 4 (35.5\%) & $9(11.1 \%)$ & $<0.001$ \\
\hline Dyslipidemia, n(\%) & $221(68.8 \%)$ & $38(46.9 \%)$ & $<0.001$ \\
\hline Smoking, $n(\%)$ & 235 (73.2\%) & $58(71.6 \%)$ & 0.772 \\
\hline Drinking, n(\%) & 209 (65.1\%) & 52 (64.2\%) & 0.878 \\
\hline Exercise, n(\%) & 228 (71.0\%) & $64(79.0 \%)$ & 0.150 \\
\hline $\mathrm{TC}(\mathrm{mmol} / \mathrm{L})$ & $3.93 \pm 1.14$ & $3.94 \pm 0.96$ & 0.945 \\
\hline $\mathrm{TG}(\mathrm{mmol} / \mathrm{L})$ & 1.39 (0.47 to 18.99$)$ & $\mathrm{I} .24(0.4 \mathrm{I}$ to 5.46$)$ & 0.136 \\
\hline LDL-c(mmol/L) & 2.24 (0.79 to 5.05$)$ & 2.31 (0.87 to 4.13$)$ & 0.962 \\
\hline $\mathrm{HDL}-\mathrm{c}(\mathrm{mmol} / \mathrm{L})$ & $0.94(0.56$ to $2.4 I)$ & 1.04 (0.63 to 1.72$)$ & 0.003 \\
\hline$L_{p}(a)(m g / L)$ & $|4|$ (I to II87) & 125 (6 to 11111$)$ & 0.183 \\
\hline Glucose (mmol/L) & 5.09 (2.98 to 15.19) & 4.68 (2.98 to 14.00$)$ & $<0.001$ \\
\hline $\mathrm{HbAlc}(\%)$ & $5.90(4.80$ to 13.00$)$ & 5.60 (4.50 to 10.70$)$ & $<0.001$ \\
\hline Family history of CAD, $n(\%)$ & 61 (19.0\%) & $17(21.0 \%)$ & 0.687 \\
\hline Severe AGA, n(\%) & $131(40.8 \%)$ & $18(22.2 \%)$ & 0.002 \\
\hline Gensini scores & 30.0 (2.0 to I52.0) & $2.0(0.0$ to 10.0$)$ & $<0.001$ \\
\hline
\end{tabular}

Abbreviations: CAD, coronary artery disease; BMI, body mass index; SBP, systolic blood pressure; DBP, diastolic blood pressure; AGA, androgenetic alopecia; TC, total cholesterol; TG, total triglyceride; HDL-c, fasting high-density lipoprotein cholesterol; LDL-c, fasting low-density lipoprotein cholesterol; Lp(a), lipoprotein(a).

Table 3 Univariate Logistic Regression Analysis with Respect to the Presence of Obstructive CAD

\begin{tabular}{|c|c|c|c|c|}
\hline Characteristic & B & OR & $95 \% \mathrm{Cl}$ & $P$ value \\
\hline Age(years) & 0.012 & 1.012 & 0.986 to 1.038 & 0.367 \\
\hline $\operatorname{BMI}\left(\mathrm{kg} / \mathrm{m}^{2}\right)$ & 0.082 & 1.085 & 1.002 to 1.176 & 0.046 \\
\hline Hypertension(yes/no) & 0.199 & 1.220 & 0.716 to 2.077 & 0.464 \\
\hline Diabetes(yes/no) & 1.483 & 4.406 & 2.124 to 9.139 & 0.000 \\
\hline Dyslipidemia(yes/no) & 0.917 & $2.50 \mathrm{I}$ & 1.523 to 4.108 & 0.000 \\
\hline Smoking(yes/no) & 0.080 & 1.084 & 0.630 to 1.864 & 0.772 \\
\hline Drinking(yes/no) & 0.040 & 1.041 & 0.626 to 1.731 & 0.878 \\
\hline Exercise(yes/no) & -0.429 & 0.651 & 0.362 to $\mathrm{I} .17 \mathrm{I}$ & 0.152 \\
\hline Family history of $C A D($ yes/no) & -0.124 & 0.883 & 0.483 to 1.614 & 0.687 \\
\hline \multicolumn{5}{|l|}{ AGA $(0 / 1 / 2)$} \\
\hline 0 (mild) & NS & 1.000 & NS & NS \\
\hline I(moderate) & -0.477 & 0.621 & 0.333 to 1.156 & 0.133 \\
\hline 2(severe) & 0.746 & 2.108 & 1.156 to 3.843 & 0.015 \\
\hline Non-severe AGA & NS & 1.000 & NS & NS \\
\hline Severe AGA & 0.881 & 2.413 & I. 366 to 4.263 & 0.002 \\
\hline Non-early-onset AGA & NS & 1.000 & NS & NS \\
\hline Early onset AGA & 0.892 & $2.44 I$ & 1.235 to 4.824 & 0.010 \\
\hline
\end{tabular}

Abbreviations: CAD, coronary artery disease; BMI, body mass index; AGA, androgenetic alopecia. 
Table 4 Multivariate Logistic Regression Analysis with Respect to the Presence of CAD

\begin{tabular}{|l|l|l|l|l|}
\hline Characteristic & B & OR & 95\% CI & P value \\
\hline AGA(0/I/2) & NS & 1.000 & NS & NS \\
\hline (mild) & -0.534 & 0.586 & 0.302 to 1.136 & 0.114 \\
I (moderate) & 0.592 & 1.807 & 0.952 to 3.430 & NS \\
2 (severe) & NS & 1.000 & I.I52 to 3.870 & NS \\
Non-severe AGA & 0.747 & 2.111 & NS & 0.070 \\
Severe AGA & NS & 1.000 & I.132 to 4.640 & NS \\
Non-early-onset AGA & 0.829 & 2.292 & 0.021 \\
Early-onset AGA &
\end{tabular}

Note: Adjusted variables: age, BMI, hypertension, diabetes, dyslipidemia, smoking status, drinking status, exercise status and family history of CAD.

Abbreviations: CAD, coronary artery disease; BMI, body mass index; AGA, androgenetic alopecia.

Table 5 Multivariate Logistic Regression Analysis with Respect to the Presence of "High Stenosis"

\begin{tabular}{|l|l|l|l|l|}
\hline Characteristic & B & OR & 95\% CI & P value \\
\hline AGA(0/I/2) & NS & 1.000 & NS & NS \\
\hline 0 (mild) & -0.185 & 0.831 & 0.457 to 1.512 & 0.545 \\
I(moderate) & 0.809 & 2.247 & 1.396 to 3.617 & 0.00 I \\
2 (severe) & NS & 1.000 & NS & NS \\
Non-severe AGA & 0.859 & 2.360 & 1.506 to 3.699 & $<0.001$ \\
Severe AGA(yes/no) & NS & 1.000 & NS & NS \\
Non-early-onset AGA & 1.245 & 3.474 & 2.069 to 5.832 & $<0.001$ \\
Early-onset AGA &
\end{tabular}

Note: Adjusted variables: age, BMI, hypertension, diabetes, dyslipidemia, smoking status, drinking status, exercise status and family history of CAD. Abbreviations: CAD, coronary artery disease; BMI, body mass index; AGA, androgenetic alopecia.

3.617, $P=0.001$ ). After adjusting for other variables, severe AGA was independently associated with "high stenosis" risk (OR, 2.360; 95\% CI 1.506 to 3.699, $P<0.001$ ). Meanwhile, the correlation between early-onset AGA and "high stenosis" also remained independent, and the relative risk of 3.474 (OR, 3.474; $95 \%$ CI 2.069 to $5.832, P<0.001$ ).

\section{The Predictive Ability of Severe AGA for Coronary Atherosclerosis Severity}

Figure 1 shows the ROC analysis of severe AGA in the diagnosis of coronary artery "high stenosis". The sensitivity of this sign to diagnose "high stenosis" is $46.9 \%$, the specificity is $73.3 \%$, the positive predictive value is $65.1 \%$, the negative predictive value is $56.5 \%$, and the Youden index is 0.202 among the subjects of this study (area under the curve $0.601,95 \%$ CI 0.546 to $0.656, P<0.001$ ).

\section{The Relationship Between AGA and CAD Risk Factors}

The underlying synergy effects of AGA with CAD risk factors have been analyzed and the results are shown in
Table 6. The results suggested that the ORs for the effects of both severe AGA exposure and obesity (BMI $>27 \mathrm{~kg} / \mathrm{m}^{2}$ ), diabetes and dyslipidemia were significantly higher than the ORs for the estimated effect of each factor in the circumstances without other factors. The proportion of the interaction of severe AGA and obesity contributing to $\mathrm{CAD}$ was $28.9 \% \quad(\mathrm{SI}=1.663, \mathrm{SIM}=1.222, \mathrm{AP}=0.289)$, and it was $50.3 \%(\mathrm{SI}=2.239, \mathrm{SIM}=1.149, \mathrm{AP}=0.503)$ for severe $\mathrm{AGA}$ and diabetes, while for severe AGA and dyslipidemia it was $4.5 \%(\mathrm{SI}=1.062, \mathrm{SIM}=0.646, \mathrm{AP}=0.045)$.

\section{Discussion}

In this study, AGA was independently and significantly associated with the increase of the prevalence of CAD in a Han Chinese male population. Participants with severe AGA or early-onset AGA had higher coronary atherosclerosis severity. Moreover, the positive correlations of severe AGA with obesity, diabetes and dyslipidemia were significantly correlated with the risk of CAD. Therefore, there is an urgent need to strengthen the management of CAD risk factors in patients with severe AGA. 


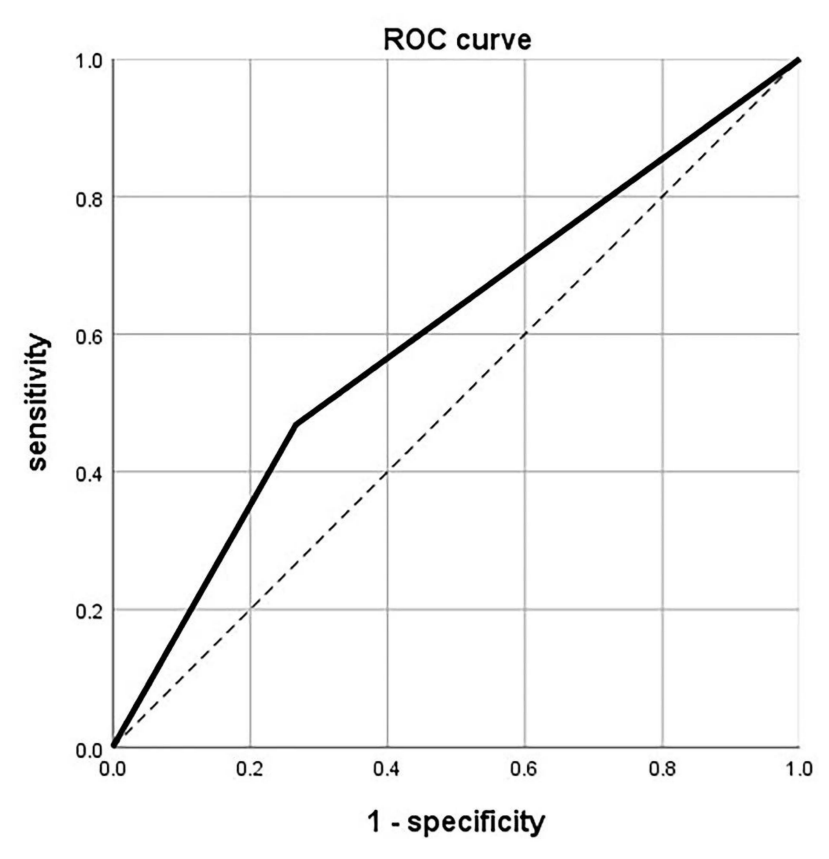

Figure I The receiver operating characteristic (ROC) curve for the "high stenosis" status of coronary arteries. The area under the curve $(A \cup C)=0.60 \mathrm{I}$; Dotted line: Reference line.

In 1972, Susan G. Cotton et al first proposed the relationship between CAD and AGA. ${ }^{24}$ Since then, several pieces of epidemiological researches have concluded that
AGA is positively relevant to coronary atherosclerosis, $\mathrm{CAD}$ and myocardial infarction. ${ }^{25-27}$ In addition, several studies have shown that early-onset AGA is associated with increased CAD risk, revealing the role of earlyonset AGA in predicting CAD risk. ${ }^{6,9}$ Some other studies have shown that alopecia, chest hair and diagonal earlobe creases can be used as dermatological indicators of CAD risk. ${ }^{28,29}$ However, another study has shown that there is no difference in myocardial infarction (MI) risk and carotid intimal-medial thickness between patients with AGA and those without AGA. AGA may not be a predictor of MI or asymptomatic atherosclerosis. ${ }^{30}$ The correlation between AGA and CAD is still controversial due to the influence of the following confounding factors: ethnic distribution, diagnostic criteria and the severity assessment for CAD (based on chest pain, subclinical vascular damage, traditional CAD risk factors, abnormal ECG at rest or CAG), and AGA diagnosis. Paulo A. Lotufo et al demonstrated that vertex AGA seems to be a sign of increased CAD risk, especially in males with hypertension or high cholesterol. ${ }^{3}$ Nevertheless, this study did not remove the interference of racial differences. Meanwhile, subjects were all physicians, whose work intensity and

Table 6 Relationship Between Severe AGA and CAD Risk Factors

\begin{tabular}{|c|c|c|c|c|c|}
\hline Classical Risk & Severe AGA & CAD & Non-CAD & OR $(95 \% \mathrm{Cl})$ & $P$ value \\
\hline \multicolumn{6}{|l|}{ Obesity } \\
\hline 0 & 0 & 135 & 48 & I & \\
\hline 0 & I & 90 & 14 & 2.286 ( $(1.190$ to 4.389$)$ & 0.013 \\
\hline 1 & 0 & 55 & 15 & I.304 (0.674 to 2.520$)$ & 0.430 \\
\hline \multirow[t]{2}{*}{1} & I & 41 & 4 & $3.644(1.240$ to $10.7 \mid 3)$ & 0.019 \\
\hline & & $\mathrm{SI}=\mathrm{I} .663$ & $\mathrm{SIM}=1.222$ & RERI $=I .054$ & $\mathrm{AP}=0.289$ \\
\hline \multicolumn{6}{|l|}{ Diabetes } \\
\hline 0 & 0 & 125 & 56 & I & \\
\hline 0 & 1 & 82 & 16 & 2.296 (1.233 to 4.275$)$ & 0.009 \\
\hline 1 & 0 & 65 & 7 & 4.160 (1.794 to 9.645$)$ & 0.001 \\
\hline 1 & I & 49 & 2 & $10.976(2.578$ to 46.727$)$ & 0.001 \\
\hline & & $\mathrm{SI}=2.239$ & $\operatorname{SIM}=1.149$ & RERI $=5.520$ & $\mathrm{AP}=0.503$ \\
\hline \multicolumn{6}{|l|}{ Dyslipidemia } \\
\hline 0 & 0 & 69 & 37 & I & \\
\hline 0 & 1 & 31 & 6 & $2.77 \mathrm{I}(1.060$ to 7.244$)$ & 0.038 \\
\hline 1 & 0 & 121 & 26 & 2.496 (1.394 to 4.467$)$ & 0.002 \\
\hline 1 & 1 & 100 & 12 & 4.469 (2.176 to 9.178$)$ & $<0.001$ \\
\hline & & $\mathrm{SI}=1.062$ & $\operatorname{SIM}=0.646$ & RERI $=0.202$ & $\mathrm{AP}=0.045$ \\
\hline
\end{tabular}

Note: $\mathrm{SI}=(\mathrm{ORII} I$ I)/(ORI0+OROI-2); SIM=ORII/(ORIO×OROI); RERI=ORII-ORIO-OROI +I; AP=RERI/ORII.

Abbreviations: CAD, coronary artery disease; AGA, androgenetic alopecia; SI, synergy measures in additive models; SIM, synergy measures in multiplicative models; AP, attributable proportion of interaction; RERI, relative excess risk of interaction. 
pressure would lead to a higher incidence of CAD and AGA. Another study revealed that hypertensive patients with severe and early-onset AGA appear to be exposed to higher CVD risk. ${ }^{6}$ Nevertheless, the major shortcoming of this study is that the severity assessment of CAD was based on subclinical vascular damage rather than $C A G$, making the results unreliable. To eliminate the distraction of ethnic differences, our subjects all consisted of Han Chinese males; they were divided into $\mathrm{CAD}$ and nonCAD groups based on the presence of CAG-certified $\mathrm{CAD}$, and AGA status is classified using the BASP classification. To our knowledge, before our study, no study has investigated the relationship between AGA and CAG in a Han Chinese male population.

In the current study, we found that AGA was independently correlated to $\mathrm{CAD}$, which is consistent with previous studies. The Gensini scoring system was used to assess the severity of coronary atherosclerosis and the participants were divided into "high stenosis" and "low stenosis" group. AGA was also found to be independently associated with "high stenosis" and to be indicative of the degree of coronary atherosclerosis. Elevated serum-free androgen levels are correlated with AGA, and may be the underlying physiological mechanism of AGA patients' susceptibility to CAD. ${ }^{11}$ In previous studies, serum-free androgens have a significant stimulating effect on DNA synthesis of VSMC and may stimulate VSMC proliferation to promote atherosclerosis and cause vasoconstriction leading to hypertension. Significant improvements in vascular endothelial function were observed in men who maintained androgen deficiency compared with those with normal androgen levels, confirming that androgen may contribute to vascular endothelial dysfunction. ${ }^{31}$ However, there are still divergent views as to whether androgens have a protective effect on CAD. Endogenous androgens may have a cardiovascular protective effect in men, reducing the risk of $\mathrm{CVD} ;{ }^{32,33}$ further research suggests that testosterone may benefit endothelial function. ${ }^{34}$ As reported by Fallah et al, ${ }^{35}$ lower levels of total testosterone have a protective effect on $\mathrm{CAD}$, while higher values increase the CAD risk. Moreover, chronic inflammation may be linked to CAD and alopecia. ${ }^{14}$ Several studies have shown that chronic inflammation of AGA hair follicles and blood vessels around hair follicles is involved in hair loss, ${ }^{14}$ and this low-level inflammation that occurs at the hair loss site may be local manifestations of systemic inflammation.
Many studies have shown that AGA is also involved in the development of CVD risk factors. ${ }^{2,12,36-38}$ It has been revealed that AGA patients have higher levels of blood pressure, LDL-C, TC, and TG. ${ }^{36}$ And AGA patients have a higher risk of metabolic syndrome. ${ }^{38}$ Hirsso et al conducted a cross-sectional study showing that AGA might be positively correlated with hypertension. ${ }^{37}$ The increase of serum aldosterone, which is an important cause of hypertension, may directly affect the development of AGA. ${ }^{2}$ Insulin resistance, another CVD risk factor, has been reported to affect the blood supply to the scalp, resulting in impaired nutrient supply to the hair follicles. ${ }^{12}$ Moreover, insulin resistance may increase free androgen levels by inhibiting SHBG (sex hormone binding globulin), ultimately leading to hair loss. ${ }^{13}$ Besides, some studies have reported that AGA is positively correlated with dyslipidemia. ${ }^{2}$ A recent study has shown that obesity is also involved in the process of AGA aggravation. Obesity can inhibit the signal transduction of Sonic hedgehog (SHH), reducing the number of hair following stem cells (HFSCs). ${ }^{39}$ In our study, patients with severe AGA had higher SBP, DBP, HbAlc, and incidence of dyslipidemia, consistent with these findings. Moreover, the positive interactions of severe AGA with obesity, diabetes and dyslipidemia were significantly correlated with the increased risk of CAD. AGA patients may need to strengthen the management of CAD risk factors, which is of great significance for the prevention and clinical diagnosis and treatment of CAD.

The superiority of our research is that it was on account of CAG and the BASP classification to diagnose CAD and AGA, respectively. Besides, in our study, the sample consisted only of the Han Chinese male population, making up for the lack of studies on the relationship between AGA and CAD in Asian people. However, several limitations exist in this study. Its sample size was small and participants were enrolled from only one hospital, which may lead to bias in clinical data. Therefore, large-scale cohort studies for the general population are of great significance. Furthermore, this cross-sectional study can only assess association rather than causation, and it is necessary to clarify it in future researches.

\section{Conclusion}

This research demonstrated that AGA is independently associated with CAD in a Han Chinese male population. AGA may be a simple and feasible method for screening CAD and indicative of the severity of coronary atherosclerosis. 


\section{Data Sharing Statement}

The data used to support the findings of this study are available from the corresponding author upon request.

\section{Ethics Approval}

Ethics Committee of the First Affiliated Hospital of Nanjing Medical University (2011-SRFA-005).

\section{Acknowledgments}

The authors thank all patients who participated in this study for their cooperation and support, and the Dermatology Department of Nanjing Medical University First Affiliated Hospital for their assistance in diagnosing and evaluating AGA.

\section{Author Contributions}

All authors made a significant contribution to the work reported, whether that is in the conception, study design, execution, acquisition of data, analysis and interpretation, or in all these areas; took part in drafting, revising or critically reviewing the article; gave final approval of the version to be published; have agreed on the journal to which the article has been submitted; and agree to be accountable for all aspects of the work.

\section{Funding}

The study did not receive any specific funding from any funding agency in the public, commercial or non-profit sectors.

\section{Disclosure}

The authors declare that there is no conflict of interest regarding the publication of this paper.

\section{References}

1. Haijiang D, Alsalhe Tariq A, Nasr C, Matteo R, Luigi BN, Jianhong W. The global burden of disease attributable to high body mass index in 195 countries and territories, 1990-2017: an analysis of the global burden of disease study. PLoS Med. 2020;17(7):e1003198. doi:10.1371/journal.pmed.1003198

2. Arias-Santiago S, Gutiérrez-Salmerón MT, Buendía-Eisman A, GirónPrieto MS, Naranjo-Sintes R. Hypertension and aldosterone levels in women with early-onset androgenetic alopecia. $\mathrm{Br} J$ Dermatol. 2010;162(4):786-789. doi:10.1111/j.1365-2133.2009.09588.x

3. Kanti V, Messenger A, Dobos G, et al. Evidence-based (S3) guideline for the treatment of androgenetic alopecia in women and in men - short version. $J$ Eur Acad Dermatol Venereol. 2018;32(1):11-22. doi: $10.1111 /$ jdv.14624

4. Lotufo PA, Chae CU, Ajani UA, Hennekens CH, Manson JE. Male pattern baldness and coronary heart disease: the physicians' health study. Arch Intern Med. 2000;160(2):165-171. doi:10.1001/ archinte.160.2.165
5. Alfredo R. Baldness and coronary artery disease: the dermatologic point of view of a controversial issue. Arch Dermatol. 2001;137 (7):943-947. doi:10-1001/pubs.ArchDermatol.-ISSN-0003-987x137-7-dre0006

6. Helen T, Agis G, Ignatios I, et al. Severity of alopecia predicts coronary changes and arterial stiffness in untreated hypertensive men. J Clin Hypertension. 2017;19(1):51-57. doi:10.1111/jch.12871

7. Danesh-Shakiba M, Jalal P, Pedram A. Androgenetic alopecia: relationship to anthropometric indices, blood pressure and life-style habits. Clin Cosmet Investig Dermatol. 2020;13:137-143. doi:10.2147/CCID.S231940

8. Sang-Yeon P, Oh SS, Lee W. Relationship between androgenetic alopecia and cardiovascular risk factors according to BASP classification in Koreans. J Dermatol. 2016;43(11):1293-1300. doi:10.1111/ 1346-8138.13355

9. Sharma KH, Jindal A. Association between androgenetic alopecia and coronary artery disease in young male patients. Int $J$ Trichology. 2014;6(1):5-7. doi:10.4103/0974-7753.136747

10. Valerie AR. Androgens and hair growth. Dermatol Ther. 2008;21 (5):314-328. doi:10.1111/j.1529-8019.2008.00214.x

11. Fujimoto R, Morimoto I, Morita E, Sugimoto H, Ito Y, Eto S. Androgen receptors, 5 alpha-reductase activity and androgen-dependent proliferation of vascular smooth muscle cells. J Steroid Biochem Mol Biol. 1994;50(3-4):169-174. doi:10.1016/ 0960-0760(94)90025-6

12. Matilainen Veikko A, Mäkinen PK, Keinänen-Kiukaanniemi SM. Early onset of androgenetic alopecia associated with early severe coronary heart disease: a population-based, case-control study. J Cardiovasc Risk. 2001;8(3):147-151. doi:10.1177/ 174182670100800305

13. Seidell Jacob C, Per B, Lars S, Henry K, Rune S. Visceral fat accumulation in men is positively associated with insulin, glucose, and C-peptide levels, but negatively with testosterone levels. Metabolism. 1990;39(9):897-901. doi:10.1016/0026-0495(90) 90297-p

14. Hirsso P, Rajala $U$, Hiltunen L, Jokelainen J, KeinänenKiukaanniemi S, Näyhä S. Obesity and low-grade inflammation among young Finnish men with early-onset alopecia. Dermatology. 2007;214(2):125-129. doi:10.1159/000098570

15. Judkins PM. Selective coronary arteriography. I. A percutaneous transfemoral technic. Radiology. 1967;89(5):815-824. doi:10.1148/ 89.5.815

16. Austen WG, Edwards JE, Frye RL, et al. A reporting system on patients evaluated for coronary artery disease. Report of the ad hoc committee for grading of coronary artery disease, Council on Cardiovascular Surgery, American Heart Association. Circulation. 1975;51(4Suppl):5-40. doi:10.1161/01.cir.51.4.5

17. Karadag B, Kucur M, Isman FK, Hacibekiroglu M, Vural VA. Serum chitotriosidase activity in patients with coronary artery disease. Circulation J. 2008;72(1):71-75. doi:10.1253/circj.72.71

18. Gensini Goffredo G. A more meaningful scoring system for determining the severity of coronary heart disease. Excerpta Med. 1983;51 (3):606. doi:10.1016/s0002-9149(83)80105-2

19. Zhang J-Z, Fan W-X, Zheng M, et al. 中国雄激素性秃发诊疗指南 [Guideline for diagnosis and treatment of androgenetic alopecia]. J Clin Dermatol. 2014;43(03):182-186. Chinese. doi:10.16761/j. cnki.1000-4963.2014.03.025

20. Won-Soo L, In RB, Phil HS, et al. A new classification of pattern hair loss that is universal for men and women: basic and specific (BASP) classification. J Am Acad Dermatol. 2006;57(1):37-46. doi:10.1016/j. jaad.2006.12.029

21. Joint Committee for Developing Chinese guidelines on Prevention and Treatment of Dyslipidemia in Adults. Chinese guidelines on prevention and treatment of dyslipidemia in adults. Chinese Circulation J. 2007;35(5):390-419. PMID: 17711682. 
22. Yang Q, Khoury Muin J. Evolving methods in genetic epidemiology. III. Gene-environment interaction in epidemiologic research. Narnia. 1997;19(1):33-43. doi:10.1093/oxfordjournals.epirev.a017944

23. Hanley AJ, Mcneil BJ. The meaning and use of the area under a receiver operating characteristic (ROC) curve. Radiology. 1982;143(1):29-36. doi:10.1148/radiology.143.1.7063747

24. Cotton SG, Nixon JM, Carpenter RG, Evans DW. Factors discriminating men with coronary heart disease from healthy controls. $\mathrm{Br}$ Heart J. 1972;34(5):458-464. doi:10.1136/hrt.34.5.458

25. Herrera CR, D’Agostino RB, Gerstman BB, Bosco LA, Belanger AJ. Baldness and coronary heart disease rates in men from the Framingham Study. Am J Epidemiol. 1995;142(8):828-833. doi:10.1093/oxfordjournals.aje.a117722

26. Schnohr P, Lange P, Nyboe J, Appleyard M, Jensen G. Gray hair, baldness, and wrinkles in relation to myocardial infarction: the Copenhagen city heart study. Am Heart J. 1995;130(5):1003-1010. doi:10.1016/0002-8703(95)90201-5

27. Mancia G, Laurent S, Agabiti-Rosei E, et al. Reappraisal of European guidelines on hypertension management: a European Society of Hypertension task force document. Blood Press. 2009;18 (6):308-347. doi:10.3109/08037050903450468

28. Mirić D, Fabijanić D, Giunio L, et al. Dermatological indicators of coronary risk: a case-control study. Int J Cardiol. 1998;67 (3):251-255. doi:10.1016/s0167-5273(98)00313-1

29. Wang Y, Mao L-H, Jia E-Z, et al. Relationship between diagonal earlobe creases and coronary artery disease as determined via angiography. BMJ Open. 2016;6(2):e008558. doi:10.1136/bmjopen2015-008558

30. Halim MM, Meyrick G, Jeans WD, Murphy D, Burton JL. Myocardial infarction, androgen and the skin. $\mathrm{Br} J$ Dermatol. 1978;98(1):63-68. doi:10.1111/j.1365-2133.1978.tb07334.x

31. Herman SM, Robinson JT, McCredie RJ, Adams MR, Boyer MJ, Celermajer DS. Androgen deprivation is associated with enhanced endothelium-dependent dilatation in adult men. Arterioscler Thromb Vasc Biol. 1997;17(10):2004-2009. doi:10.1161/01.atv.17.10.2004
32. Yeap $\mathrm{Bu}$ B. Androgens and cardiovascular disease. Curr Opin Endocrinol Diabetes Obes. 2010;17(3):269-276. doi:10.1097/ MED.0b013e3283383031

33. Akishita M, Hashimoto M, Ohike Y, et al. Low testosterone level as a predictor of cardiovascular events in Japanese men with coronary risk factors. Atherosclerosis. 2010;210(1):232-236. doi:10.1016/j. atherosclerosis.2009.10.037

34. Akishita M, Hashimoto M, Ohike Y, et al. Low testosterone level is an independent determinant of endothelial dysfunction in men. Hypertension Res. 2007;30(11):1029-1034. doi:10.1291/ hypres.30.1029

35. Fallah N, Mohammad K, Nourijelyani K, et al. Nonlinear association between serum testosterone levels and coronary artery disease in Iranian men. Eur J Epidemiol. 2009;24(6):297-306. doi:10.1007/ s10654-009-9336-9

36. Sharma L, Dubey A, Gupta PR, Agrawal A. Androgenetic alopecia and risk of coronary artery disease. Indian Dermatol Online J. 2013;4 (4):283-287. doi:10.4103/2229-5178.120638

37. Päivi H, Mauri L, Veikko M, et al. Association of insulin resistance linked diseases and hair loss in elderly men. Finnish population-based study. Cent Eur J Public Health. 2006;14(2):78-81. doi:10.21101/ cejph.b0045

38. Arias-Santiago S, Gutiérrez-Salmerón MT, Castellote-Caballero L, Buendía-Eisman A, Naranjo-Sintes R. Alopecia androgénica masculina y factores de riesgo cardiovascular: estudio de casos y controles [Male androgenetic alopecia and cardiovascular risk factors: a casecontrol study]. Actas Dermosifiliogr. 2010;101(3):248-256. PMID: 20398601. doi:10.1016/j.ad.2009.10.002

39. Morinaga H, Mohri Y, Grachtchouk M, et al. Obesity accelerates hair thinning by stem cell-centric converging mechanisms. Nature. 2021;595(7866):266-271. doi:10.1038/s41586-021-03624-x
International Journal of General Medicine

\section{Publish your work in this journal}

The International Journal of General Medicine is an international, peer-reviewed open-access journal that focuses on general and internal medicine, pathogenesis, epidemiology, diagnosis, monitoring and treatment protocols. The journal is characterized by the rapid reporting of reviews, original research and clinical studies

\section{Dovepress}

across all disease areas. The manuscript management system is completely online and includes a very quick and fair peer-review system, which is all easy to use. Visit http://www.dovepress.com/ testimonials.php to read real quotes from published authors. 